\title{
Tracer dispersion in bedload transport
}

\section{E. Lajeunesse, O. Devauchelle, M. Houssais, and G. Seizilles}

Institut de Physique du Globe - Sorbonne Paris Cité, Équipe de Dynamique des fluides géologiques, 1 rue Jussieu, 75238 Paris cedex 05, France

Correspondence to: E. Lajeunesse (lajeunes@ipgp.fr)

Received: 26 February 2013 - Revised: 25 June 2013 - Accepted: 1 July 2013 - Published: 17 December 2013

\begin{abstract}
Bedload particles entrained by rivers tends to disperse as they move downstream. In this paper, we use the erosion-deposition model of Charru et al. (2004) to describe the velocity and the spreading of a plume of tracer particles. We restrict our analysis to steady-state transport above a flat bed of uniform sediment. The transport of tracer particles is then controlled by downstream advection and particle exchange with the immobile bed. After a transitional regime dominated by initial conditions, the evolution of a plume of markers tends asymptotically towards classical advection-diffusion: its average position grows linearly with time, whereas it spreads like the square root of time.
\end{abstract}

\section{Introduction}

Bedload transport results from the entrainment of a granular bed by a flow. Superficial grains are set into motion by shear stress and move by rolling, by sliding or by a succession of low jumps above the sediment bed. Eventually, the entrained grains settle back on the bed. Bedload transport thus results from the continuous exchange of particles between the bed and the layer of moving grains (the bedload layer).

Bedload particles disperse as they move downstream. While numerous studies focus on the sediment transport rate at a specific location (Shields, 1936; Meyer-Peter and Müller, 1948; Einstein, 1950; Ancey et al., 2008), the velocity and dispersion of individual particles has received much less attention. In other words, most studies favor the Eulerian point of view. Yet, the Lagragian approach is required for many applications, such as the transport of solid-phase contaminants in streams (Sayre and Hubbell, 1965; Cerling et al., 1990; Hassan and Ergenzinger, 2003; Bradley et al., 2010), or the accumulation of cosmogenic radionuclides in sediment



Fig. 1. A granular bed sheared by a steady and uniform flow. The sediment bed is a mixture of marked (in grey) and unmarked grains.

grains during their transport (Carretier et al., 2007; Gayer et al., 2008; Willenbring and von Blanckenburg, 2010).

Tracer particles have thus been used, both in the field and in laboratory experiments, to quantify bedload entrainment at a fixed location (Reid et al., 1985; Wilcock, 1997), the travel distance of a single particle (Ferguson and Wathen, 1998; Martin et al., 2012), or particle storage in the sediment bed (Haschenburger and Church, 1998). Most studies revealed right-skewed and fat-tailed distributions of the travel distance. These findings are usually interpreted as the signature of superdiffusion in bedload (Habersack, 2001; McNamara and Borden, 2004; Schmidt and Ergenzinger, 2006; Bradley et al., 2010; Martin et al., 2012).

Here we use the erosion-deposition model of Charru et al. (2004) to describe the average velocity and the spreading of a plume of tracer particles. We restrict our analysis to steadystate transport above a flat bed of uniform sediment. This work was presented at the workshop SALADYN, Institut de Physique du Globe de Paris, France, 5-7 November 2012.

\section{The erosion-deposition model}

We consider a bed of particles of uniform size $d_{s}$ and density $\rho_{s}$ sheared by a fluid of density $\rho$ and kinematic viscosity $v$. 
Table 1. Comparison of sediment transport characteristics for laminar and turbulent flows: particle settling velocity $V_{S}$, mean streamwise velocity of the particles $V$, erosion time $t_{e}$, and settling time $t_{s}$.

\begin{tabular}{ccc}
\hline & $\begin{array}{c}\text { Laminar flow } \\
\text { Charru (2006) }\end{array}$ & $\begin{array}{c}\text { Turbulent flow } \\
\text { Lajeunesse et al. (2010) }\end{array}$ \\
\hline$V_{s}$ & $\frac{\left(\rho_{s} / \rho-1\right) g d_{s}^{2}}{18 v}$ & $\sqrt{\left(\rho_{s} / \rho-1\right) g d_{s}}$ \\
$\frac{V}{V_{s}}$ & $1.8 \theta$ & $4.4\left(\theta^{1 / 2}-\theta_{t}^{1 / 2}\right)+0.11$ \\
$t_{e}$ & $33 \frac{d_{s}}{V_{s}}$ & $2 \frac{\rho_{s}}{\rho} \frac{d_{s}}{V_{s}}$ \\
$t_{s}$ & $15 \frac{d_{s}}{V_{s}}$ & $11 \frac{d_{s}}{V_{s}}$ \\
\hline
\end{tabular}

The flow, either laminar or turbulent, applies a shear stress $\tau$ on the sediment bed. The bedload transport rate reads

$q_{\mathrm{s}}=n V$,

where $n$ is the surface concentration of entrained particles, i.e. the number of particles in motion per unit bed area, and $V$ is the mean particle velocity.

At equilibrium, the particle mass balance reads (Fig. 1)

$\frac{\partial n}{\partial t}+V \frac{\partial n}{\partial x}=\dot{n}_{e}-\dot{n}_{d}$

where $x, t, \dot{n}_{e}$ and $\dot{n}_{d}$ are the streamwise coordinate, time, the number of bed particles set in motion per unit time and surface (erosion rate), and the number of particles settling on the sediment bed per unit time and surface (deposition rate), respectively.

Mass balance needs to be complemented with expressions for the particles fluxes. We use simple expressions based on particle-tracking experiments (Charru, 2006; Lajeunesse et al., 2010).

At first order, the deposition rate is proportional to the surface concentration of entrained particles:

$\dot{n}_{d}=\frac{n}{t_{s}}$

where $t_{s}$ is the characteristic settling time of a particle, assuming its elevation is about a grain diameter (Table 1). Similarly, the erosion rate is inversely proportional to the concentration of particles at the bed surface (about $1 / d_{s}^{2}$ ). Erosion occurs only if the shear stress $\tau$ exceeds a threshold value, expressed in terms of the Shields parameter $\theta=\tau /\left(\rho_{s}-\rho\right) g d_{s}$ (Shields, 1936). Finally, the erosion rate reads

$\dot{n}_{e}=\frac{\sigma}{t_{e}}\left(\theta-\theta_{t}\right)$ where $\sigma \sim 1 / d_{s}^{2}, \theta_{t}$, and $t_{e}$ are the number of immobile grains per unit bed surface, the threshold Shields parameter and the erosion time scale respectively (table 1).

The average streamwise velocity of the particles $V$ depends on the flow conditions (Table 1). It is proportional to the Shields stress in a viscous flow (Charru et al., 2004), whereas it increases as the square root of the Shields stress in a turbulent flow (Lajeunesse et al., 2010). However, this does not alter the qualitative behavior of the erosion-deposition model (table 1).

In the rest of the paper, we focus on the case of steady state uniform sediment transport. In this configuration, erosion and deposition balance each other so that Eqs. (2), (3) and (4) reduce to

$\dot{n}_{d}=\dot{n}_{e}=\frac{n}{t_{s}}$

The surface concentration of moving particles, $n$, is thus constant with respect to both time and space,

$\frac{n}{\sigma}=\frac{t_{s}}{t_{e}}\left(\theta-\theta_{t}\right)$.

This relation, well verified empirically, can also be interpreted as the consequence of stress partition between the fluid and the bed particles (Bagnold, 1954; Durán et al., 2012).

\section{Transport of a plume of tracers}

We now study how a plume of passive tracer particles is entrained by bedload transport. To do so, we separate the particles in two categories with the same physical properties, hereafter referred to as "marked" and "unmarked" (Fig. 1). We furthermore assume that sediment transport is uniform and steady. In this configuration, the Shields stress, and consequently the mean particle velocity, the erosion and deposition rates and the surface concentration of moving particles are constant. As a result, the problem is independent of the flow type (turbulent or laminar). More generally, the physical mechanism at the origin of longitudinal dispersion is the exchange of tracer particles between a static layer and a moving layer. Thus, although the model is presented in the context of bedload transport, it applies to any steady uniform system in which a passive tracer is exchanged between two layers moving at different velocities.

Let us define $\phi$ as the proportion of marked grains in the moving layer. Similarly, $\psi$ is the proportion of marked grains on the bed surface. The concentration of marked grains, defined as the ratio of the number of marked particles to the total number of particles, is

$c=n \phi+\sigma \psi=\frac{1}{\alpha+1} \psi+\frac{\alpha}{\alpha+1} \phi$

where we introduce $\alpha=n / \sigma$, the ratio of the surface concentration of moving particles to the concentration of static 


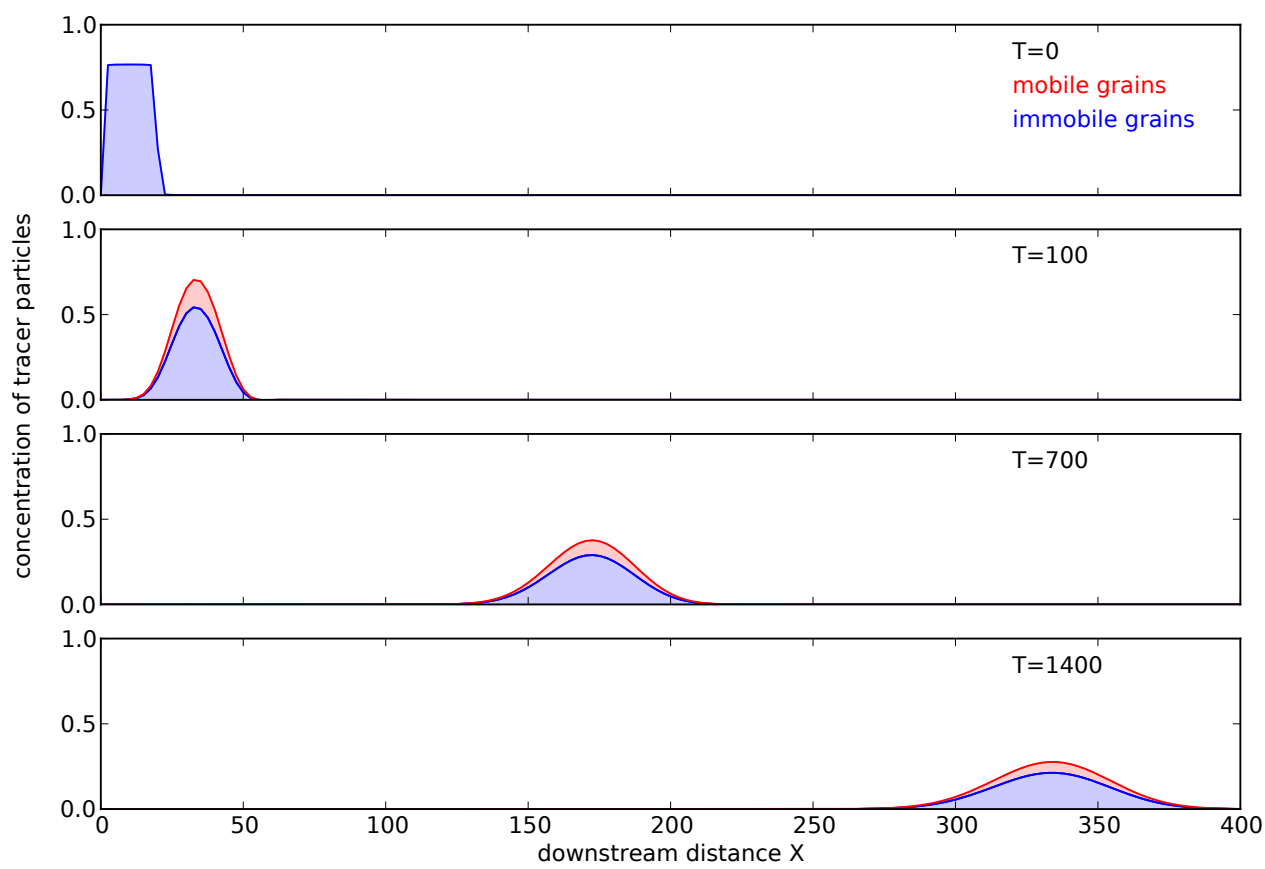

Fig. 2. Evolution of the concentration of tracer particles as a function of the dimensionless distance $X$, for $\alpha=0.3$. The initial shape of the plume is a step of marked grains on the bed surface of length 20 .

particles (this ratio is smaller than one). The total amount of tracers, $M=\int c \mathrm{~d} x$, is conserved.

The conservation equation for marked grains in the moving layer reads

$\frac{\partial}{\partial t}(\phi n)=-\frac{\partial}{\partial x}(\phi n V)+\dot{n}_{e} \psi-\dot{n}_{d} \phi$

Combining Eqs. (5) and (8), we find the evolution equation for the proportion of marked grains in the moving layer:

$\frac{\partial \phi}{\partial t}=\frac{1}{t_{s}}(\psi-\phi)-V \frac{\partial \phi}{\partial x}$.

We now assume that the "active" part of the bed is confined to the bed surface. In other words, erosion and deposition only modify the bed over a depth of about one grain diameter. Mathematically, this means that the reservoir of immobile grains is well-mixed. The conservation of marked grains on the bed surface then reads

$\sigma \frac{\partial \psi}{\partial t}=\dot{n}_{d} \phi-\dot{n}_{e} \psi$

Combining Eqs. (5) and (10) leads to

$\frac{\partial \psi}{\partial t}=-\frac{\alpha}{t_{s}}(\psi-\phi)$

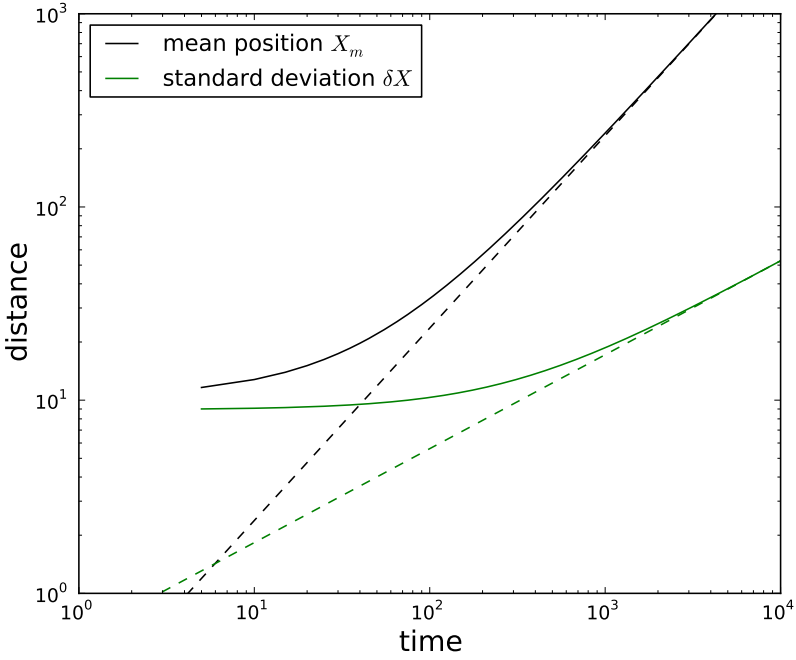

Fig. 3. Mean position $X_{m}$ (black line) and standard deviation $\delta X$ (green line) of a plume of tracers as a function of time for $\alpha=0.3$. Dashed lines show the power laws expected for standard advectiondiffusion: linear (black) and square root (green).

Complemented with initial and boundary conditions, Eqs. (9) and (11) represent the evolution of the proportion of marked particles. 


\section{Numerical solution}

In dimensionless form, Eqs. (9) and (11) read

$$
\begin{aligned}
& \frac{\partial \phi}{\partial T}=-\frac{\partial \phi}{\partial X}+(\psi-\phi) \\
& \frac{\partial \psi}{\partial T}=-\alpha(\psi-\phi) .
\end{aligned}
$$

where $T=t / t_{s}$ and $X=x t_{s} / V$ are dimensionless variables. Our system is then controlled by a single parameter, the ratio of surface densities $\alpha$. This ratio also characterize the average distance between moving grains. Since our model assumes that the behavior of an individual particle is independent from other particles, we can only expect it to be valid when moving particles are sufficiently far away from each other, that is, when $\alpha$ is small. After Eq. (4), this requirement implies that the Shields parameter be near the threshold for sediment transport.

We solve Eqs. (12) and (13) numerically with a finitevolume numerical scheme described in appendix A. An initially concentrated plume of tracer particles is transported downstream by the flow and deformed by dispersion (Fig. 2). Initially, the plume is skewed towards the direction of its propagation. This skewness, however, decreases as the plume spreads, and the plume's shape resemble a normal distribution at long times.

As expected, the average position of the plume $X_{m}=$ $\int c x \mathrm{~d} x / M$ increases with time (Fig. 3). After a transitional period of moderate velocity, dominated by initial conditions, it reaches a permanent regime with a constant average velocity. The standard deviation of the plume $\delta X=$ $\sqrt{\int c\left(x-X_{m}\right)^{2} \mathrm{~d} x} / M$ increases as the square root of time, again after a transitional regime. This behavior corresponds to classical advection-diffusion, although we have not formally established this equivalence. We observe the same behavior for various values of $\alpha$.

\section{Conclusions}

Based on an erosion-deposition model, we describe the evolution of a plume of tracer particles transported as bedload over a uniform bed. The mean position of the plume, as well as its extent, show two regimes: (i) at short times, the evolution of the plume is dictated by the initial conditions; (ii) at long times, the plume evolves asymptotically as if transported by advection and spread by diffusion.

In the model presented here, the spread of the plume results only from the continuous exchange of particles between the bedload layer and the immobile bed. Although presented in the context of bedload transport, it applies to any steady uniform system in which some passive tracer is exchanged between two layers moving at different velocities. This mechanism can be thought of as a simpler version of Taylor diffusion, where a passive tracer diffuses in a Poiseuille flow (Taylor, 1953). In our case, the distribution of velocities is reduced to the two end-members of the Poiseuille profile: mobile or immobile. It is therefore not surprising to find similar results.

In reality, the distribution of particle velocities in bedload transport is continuous (Lajeunesse et al., 2010; Furbish et al., 2012a, b). Therefore, moving grains in the bedload layer also disperse due to velocity fluctuations. This effect, adding to the continuous exchange of particles between the bedload layer and the immobile bed, is likely to enhance the dispersion of the plume of tracers.

\section{Appendix A}

\section{Numerical Scheme}

In this section, we briefly present the numerical scheme used to solve Eqs. (12) and (13).

Let us define $\phi_{k}(T)$ and $\psi_{k}(T)$ as the values of $\phi(X, T)$ and $\psi(X, T)$ at the $K$ discrete locations $X_{k}, k=0, \cdots, K-1$ and at time $T$. Integrating (12) between $X_{k}$ and $X_{k}+1$ leads to

$$
\int_{X_{k}}^{X_{k+1}} \frac{\partial \phi}{\partial T} d X=-\int_{X_{k}}^{X_{k+1}} \frac{\partial \phi}{\partial X} d X+\int_{X_{k}}^{X_{k+1}}(\psi-\phi) d X
$$

Discretizing the integrals gives the following $(K-1)$ equations defined for $k=0, \ldots, K-2$ :

$$
\begin{aligned}
\frac{\partial}{\partial T}\left[\delta X_{k+1}\left(\phi_{k+1}+\phi_{k}\right)\right]= & -\phi_{k+1}+\phi_{k}-\delta X_{k+1}\left(\phi_{k+1}+\phi_{k}\right) \\
& +\delta X_{k+1}\left(\psi_{k+1}+\psi_{k}\right)
\end{aligned}
$$

where we define $\delta X_{k+1}=\left(X_{k+1}-X_{k}\right) / 2$.

The boundary condition, $\phi(X=0, T)=0$, provides the $K^{- \text {th }}$ equation:

$\phi_{0}=0$

The above equations can be written in the vectorial form

$$
\frac{\partial}{\partial T} A \boldsymbol{\phi}=(-A+B+C) \boldsymbol{\phi}+A \boldsymbol{\psi}
$$

where we introduce the vectors

$\boldsymbol{\phi}=\left(\begin{array}{c}\phi_{0} \\ \vdots \\ \phi_{K-1}\end{array}\right)$ and $\boldsymbol{\psi}=\left(\begin{array}{c}\psi_{0} \\ \vdots \\ \psi_{K-1}\end{array}\right)$

and the matrixes

$A=\left(\begin{array}{cccccc}1 & \ldots & \ldots & \ldots & \ldots & 0 \\ \delta X_{1} & \delta X_{1} & & & & \vdots \\ \vdots & \ddots & \ddots & & & \vdots \\ \vdots & & \delta X_{k} & \delta X_{k} & & \vdots \\ \vdots & & & \ddots & \ddots & 0 \\ 0 & \ldots & \ldots & \ldots & \delta X_{K-1} & \delta X_{K-1}\end{array}\right)$, 
$B=\left(\begin{array}{cccc}0 & \ldots & \ldots & 0 \\ 1 & -1 & & \vdots \\ \vdots & \ddots & \ddots & 0 \\ 0 & \ldots & 1 & -1\end{array}\right)$ and $C=\left(\begin{array}{cccc}1 & 0 & \ldots & 0 \\ 0 & 0 & & \vdots \\ \vdots & & \ddots & \vdots \\ 0 & \ldots & \ldots & 0\end{array}\right)$

Similarly, discretizing (13) leads to the set of $K$ equations defined for $k=0, \ldots, K-1$

$\frac{\partial}{\partial T} \psi_{k}=\alpha\left(\phi_{k}-\psi_{k}\right)$

which can be written in the vectorial form

$\frac{\partial}{\partial T} I \psi=\alpha I(\phi-\psi)$

where $I$ is the identity matrix.

The set of Eqs. (A1) and (A2) can be combined into

$\frac{\partial}{\partial T} M_{1} \boldsymbol{\Phi}=M_{2} \boldsymbol{\Phi}$

where we define the vector

$\Phi=\left(\begin{array}{c}\phi \\ \psi\end{array}\right)$

and the matrixes

$M_{1}=\left(\begin{array}{ll}A & 0 \\ 0 & I\end{array}\right), M_{2}=\left(\begin{array}{cc}-A+B+C & A \\ \alpha I & -\alpha I\end{array}\right)$.

Finally, (A3) leads to

$\frac{\partial}{\partial T} \boldsymbol{\Phi}=M_{3} \boldsymbol{\Phi}$

with

$M_{3}=M_{1}^{-1} M_{2}$

Let us define $\boldsymbol{\Phi}^{n}$ as the value of the vector $\boldsymbol{\Phi}$ at the discrete time $T_{n}, n=0, \cdots, N$. Integrating (A4) between $T_{n}$ and $T_{n+1}$ leads to :

$$
\int_{T_{n}}^{T_{n+1}} \frac{\partial}{\partial T} \boldsymbol{\Phi} d T=\int_{T_{n}}^{T_{n+1}} M_{3} \boldsymbol{\Phi} d T
$$

which, after discretizing the integrals, reads

$\boldsymbol{\Phi}^{n+1}-\boldsymbol{\Phi}^{n}=\delta T^{n+1} M_{3}\left(\boldsymbol{\Phi}^{n+1}+\boldsymbol{\Phi}^{n}\right)$

where we introduce $\delta T^{n+1}=\left(T_{n+1}-T_{n}\right) / 2$. After some rearrangement, we finally get

$\boldsymbol{\Phi}^{n+1}=M_{4} \boldsymbol{\Phi}^{n}$

with

$M_{4}=\left(I-\delta T^{n+1} M_{3}\right)^{-1}\left(I+\delta T^{n+1}{ }_{3}\right)$

Equation (A5) enables the computation of $\boldsymbol{\Phi}^{n}$ at all times $T_{n}, n>0$ once the initial condition $\boldsymbol{\Phi}^{0}$ is known.
Acknowledgements. We would like to thank Eric Gayer, Douglas Jerolmack and Philippe Claudin for fruitful discussions.

Edited by: F. Métivier and Z. Dong

Reviewed by: two anonymous referees

\section{References}

Ancey, C., Davison, A., Bohm, T., Jodeau, M., and Frey, P.: Entrainment and motion of coarse particles in a shallow water stream down a steep slope, J. Fluid Mech., 595, 83-114, doi:10.1017/S0022112007008774, 2008.

Bagnold, R.: Experiments on a gravity-free dispersion of large solid spheres in a Newtonian fluid under shear, P. Roy. Soc. A-Math. Phy., 49-63, 1954.

Bradley, D. N., Tucker, G. E., and Benson, D. A.: Fractional dispersion in a sand bed river, J. Geophys. Res., 115, F00A09, doi:10.1029/2009JF001268, 2010.

Carretier, S., Regard, V., and Soual, C.: In situ cosmogenic nuclides in river bedload. Implications for catchment-scale erosion rate and surface exposure dating, Geochim. Cosmochim. Ac., 71, A147-A147, 2007.

Carretier, S., Regard, V., and Soual, C.: Theoretical cosmogenic nuclide concentration in river bed load clasts: Does it depend on clast size?, Quat. Geochronol., 4, 108-123, 2009.

Cerling, T. E., Morrison, S. J., Sobocinski, R. W., and Larsen, I. L.: Sediment-water interaction in a small stream: Adsorption of 137Cs by bed load sediments, Water Resour. Res., 26, 11651176, 1990.

Charru, F.: Selection of the ripple length on a granular bed sheared by a liquid flow, Phys. Fluids, 18, 121508-1-121508-9, doi:10.1063/1.2397005, 2006.

Charru, F., Mouilleron, H., and Eiff, O.: Erosion and deposition of particles on a bed sheared by a viscous flow, J. Fluid Mech., 519, 55-80, 2004.

Durán, O., Andreotti, B., and Claudin, P.: Numerical simulation of tubulent sediment transport, from bed load to saltation, Phys. Fluids, 24, 2012.

Einstein, H.: The bed-load function for sediment transportation in open channel flows, US Department of Agriculture, Soil Conservation Service, 1950.

Ferguson, R. and Wathen, S.: Tracer-pebble movement along a concave river profile: Virtual velocity in relation to grain size and shear stress, Water Resour. Res., 34, 2031-2038, 1998.

Furbish, D., Ball, A., and Schmeeckle, M.: A probabilistic description of the bed load sediment flux: 4. Fickian diffusion at low transport rates, J. Geophys. Res., 117, F03034, doi:10.1029/2012JF002356, 2012a.

Furbish, D., Roseberry, J., and Schmeeckle, M.: A probabilistic description of the bed load sediment flux: 3 . The particle velocity distribution and the diffusive flux, J. Geophys. Res., 117, F03033, doi:10.1029/2012JF002355, 2012b.

Gayer, E., Mukhopadhyay, S., and Meade, B. J.: Spatial variability of erosion rates inferred from the frequency distribution of cosmogenic ${ }^{3} \mathrm{He}$ in olivines from Hawaiian river sediments, Earth Planet. Sci. Lett., 266, 303-315, 2008.

Habersack, H.: Radio-tracking gravel particles in a large braided river in New Zealand: A field test of the stochastic theory of bed 
load transport proposed by Einstein, Hydrol. Process. 15, 377391, 2001.

Haschenburger, J. K. and Church, M.: Bed material transport estimated from the virtual velocity of sediment, Earth Surf. Proc. Land., 23, 791-808, 1998.

Hassan, M. A. and Ergenzinger, P.: Use of tracers in fluvial geomorphology, Tools in fluvial geomorphology, 397-423, 2003.

Lajeunesse, E., Malverti, L., and Charru, F.: Bedload transport in turbulent flow at the grain scale: experiments and modeling, J. Geophys. Res. Earth Surf., 115, F04001, doi:10.1029/2009JF001628, 2010.

Martin, R. L., Jerolmack, D. J., and Schumer, R.: The physical basis for anomalous diffusion in bed load transport, J. Geophys. Res., 117, F01018, doi:10.1029/2011JF002075, 2012.

McNamara, J. P. and Borden, C.: Observations on the movement of coarse gravel using implanted motion-sensing radio transmitters, Hydrol. Process., 18, 1871-1884, 2004.

Meyer-Peter, E. and Müller, R.: Formulas for Bed-Load Transport, in: Proceedings, 2nd Congress, International Association of Hydraulic Research, edited by: Stockholm, S., 39-64, 1948.
Reid, I., Frostick, L. E., and Layman, J. T.: The incidence and nature of bedload transport during flood flows in coarse-grained alluvial channels, Earth Surf. Proc. Land., 10, 33-44, 1985.

Sayre, W. and Hubbell, D.: Transport and dispersion of labeled bed material, North Loup River, Nebraska, Tech. Rep. 433-C, U.S. Geol. Surv. Prof. Pap., 1965.

Schmidt, K.-H. and Ergenzinger, P.: Bedload entrainment, travel lengths, step lengths, rest periods - Studied with passive (iron, magnetic) and active (radio) tracer techniques, Earth Surf. Proc. Land., 17, 147-165, 2006.

Shields, A. S.: Anwendung der Aehnlichkeitsmechanik und der Turbulenzforschung auf die Geschiebebewegung, Mitteilung der Preussischen Versuchsanstalt für Wasserbau und Schiffbau, 26, 1936.

Taylor, G.: Dispersion of soluble matter in solvent flowing slowly through a tube, P. Roy. Soc. A-Math. Phy., 219, 186-203, 1953.

Wilcock, P. R.: Entrainment, displacement and transport of tracer gravels, Earth Surf. Proc. Land., 22, 1125-1138, 1997.

Willenbring, J. K. and von Blanckenburg, F.: Meteoric cosmogenic Beryllium-10 adsorbed to river sediment and soil: Applications for Earth-surface dynamics, Earth Sci. Rev., 98, 105-122, 2010. 